\title{
Desafíos para la preparación de docentes de matemáticas en la tercera década del siglo
}

\section{Challenges for mathematics teacher preparation in the third decade of this century}

\section{Desafios para a preparação dos professores de matemática na terceira década do século}

\author{
Ángel Ruiz \\ Universidad de Costa Rica \\ San José, Costa Rica \\ ruizz.angel@gmail.com \\ (D) https://orcid.org/0000-0002-6481
}

Recibido - Received - Recebido: 25 / 03 / 2021 Aceptado - Accepted - Aprovado: 30 / 04 / 2021

DOI: https://doi.org/10.22458/ie.v23i34.3516

URL: https://revistas.uned.ac.cr/index.php/innovaciones/article/view/3516

\begin{abstract}
Resumen: Se plantea la urgencia de una transformación de los programas de formación de docentes que enseñan matemáticas en Costa Rica. Para sostener esta postura se analiza la situación de la Reforma Matemática de Costa Rica que comenzó oficialmente en el 2012. Se enfatiza los currículos nacionales desde la segunda mitad del siglo XX, y en particular, se señalan los de 1964 y 1995; en el primer caso un currículo dominado por la reforma de las Matemáticas Modernas (New Math), y en el segundo, programas que se declararon formalmente "constructivistas". Se afirma que en el 2012 se dio una "puntilla" final a la New Math pero, además, una ruptura con el conductismo y otros "paradigmas" educativos, en convergencia con investigación y experiencia en la Educación Matemática internacional. La crisis educativa nacional desde el 2020 ha replanteado condiciones y ritmos para la Reforma Matemática, en particular debido al grave retroceso de la escolaridad. Aquí es donde se reclama un ajuste en la formación inicial docente.
\end{abstract}

Palabras Clave: Formación de docentes, Matemáticas, Currículo, Reforma matemática, Costa Rica, Educación

Summary: This work raises the urgency for transforming the training programs for teachers who teach Mathematics in Costa Rica. In order to support this contention, the situation of the Costa Rican Mathematics Reformation that began in 2012 is analyzed. National curricula from the second half of the 20th century are emphasized, and in particular, those of years 1964 and 1995 are pointed out. Firstly, we consider curricula dominated by the Reform of Modern Mathematics New Math), and secondly, programs that were formally declared "constructivist." It is stated that in 2012 a final "coup de grace" was given to the New Math but, in addition, along with a break with behaviorism and other educational "paradigms" in convergence with research and experience in international Mathematics Education. The national educational crisis since 2020 has rethought conditions and rhythms for the Mathematics Reform, in particular, due to the serious decline in schooling. Here is where an adjustment in initial teacher training is needed.

Keywords: teacher preparation, mathematics, curriculum, Mathematics reform, Costa Rica, education 
Resumo: A urgência de uma transformação dos programas de formação de professores que ensinam matemática na Costa Rica é levantada. Para apoiar esta posição, é analisada a situação da Reforma Matemática na Costa Rica, que começou oficialmente em 2012. Destacam-se os currículos nacionais desde a segunda metade do século XX e, em particular, os de 1964 e 1995; no primeiro caso, um currículo dominado pela reforma da Matemática Moderna (Nova Matemática), e no segundo, programas que se declararam formalmente "construtivistas". Afirma-se que em 2012 foi dado um "ponto" final à Nova Matemática, mas, além disso, uma ruptura com o comportamentalismo e outros "paradigmas" educacionais, em convergência com a pesquisa e a experiência em Educação Matemática internacional. A crise educacional nacional desde 2020 redefiniu as condições e ritmos para a Reforma Matemática, particularmente devido ao grave atraso escolar. É aqui que é necessário um ajuste na formação inicial de professores.

Palavras chave: formação de professores, matemática, currículo, Reforma da Matemática, Costa Rica, educação

\section{INTRODUCCIÓN}

Es relevante visualizar el lugar histórico de los programas de formación de docentes que enseñan matemáticas. Sin duda, los enfoques curriculares precisos hacia áreas o contenidos matemáticos son muy importantes, al igual lo es comprender la urgencia de avances en las didácticas específicas en las matemáticas, o potenciar la consistencia global de la práctica docente y administrativo-docente con este currículo. No obstante, todos estos asuntos deben inscribirse en el escenario general donde se encuentra la Reforma Matemática de Costa Rica en mitad de una crisis nacional con múltiples implicaciones, se requiere calibrar la etapa histórica que se atraviesa, redefinir prioridades y, en particular, identificar desafíos novedosos para las universidades.

Cuando en el 2012 se aprobaron nuevos programas de estudio en matemáticas para el Ministerio de Educación Pública de Costa Rica, los diversos agentes educativos sabían que su implementación tomaría mucho tiempo, era una decisión audaz y estratégica por varias razones. Una, era porque el nuevo currículo busca compensar muchos años de atraso en la Educación Matemática que se daban por múltiples factores (MEP, 2012, pp. 480-485). Otra, porque se apostó a un cambio profundo y no a una colección de parches remediales: una propuesta ambiciosa que, si bien se anclaba en la realidad nacional, también quería empujarla hacia adelante. De entrada, había variables nacionales que debían transformarse para poder lograrse una implementación curricular "estable" y había que superar perspectivas y diseños educativos anteriores que todavía generaban obstáculos (Ruiz, 2020).

\section{DESARROLLO}

Los currículos nacionales dentro de un sistema educativo centralizado, como el costarricense, han sido siempre decisivos para la enseñanza y el aprendizaje. Por eso, resulta importante hacer una reflexión histórica sobre los de Matemáticas, y así ofrecer una perspectiva amplia.

En la segunda mitad del siglo XX se dieron dos importantes momentos: el primero, Barrantes y Ruiz (1995) reportan un cambio muy drástico en 1964 con un nuevo currículo, el cual tenía fuerte influencia de la reforma de las Matemáticas Modernas (New Math) en las décadas de 1960 y 1970. Aquella reforma internacional apuntaba a introducir una colección de ideas y contenidos en la secundaria y la primaria: teoría de conjuntos y estructuras algebraicas más o menos en todos los niveles, y, cual premisa, un debilitamiento (casi exclusión) de la geometría euclidiana. Basta recordar el grito de guerra "Euclides debe irse" (o "Abajo Euclides") esgrimido por Jean Dieudonné (del grupo Bourbaki) en un influyente seminario celebrado en Royaumont (Francia) en 1959. En la definición del programa de 1964 en Costa Rica fue central la participación de B. Alfaro-Sagot (director en ese momento del Departamento de Física y Matemática de la Universidad de Costa Rica) en la I CIAEM (First Inter-American Conference on Mathematical Education), Bogotá, diciembre 1961, gestada y liderada por el matemático estadounidense 
M. Stone. Alfaro-Sagot dirigió la comisión que diseñó los nuevos programas (Barrantes y Ruiz, 1995). Esa reforma encerraba muchas dificultades para la enseñanza de las matemáticas. La comunidad internacional se distanció de esta desde la segunda mitad de los años 1970; no obstante, Costa Rica duró mucho en desligarse de sus ideas.

En Costa Rica la influencia de la New Math se dio desde 1964 hasta 1995, cuando se adoptó formalmente una perspectiva "constructivista" en un nuevo currículo, aunque muy general (MEP, 2012). En ese sentido, 1995 constituyó otro momento importante para los programas de matemáticas. No obstante, en todo el siglo, incluyendo los programas de 1964 y 1995, por una razón u otra, se concebían los listados de contenidos como "lo importante". Ruiz (2018) añade aquí la influencia durante décadas de una visión conductista que atomiza y desarticula contenidos, objetivos, metodologías, evaluación, una "camisa de fuerza" para los objetos curriculares (pp. 213-214).

Ya en el siglo XXI, el currículo se separó drásticamente del conductismo y la visión curricular de los listados de contenidos. El MEP (2012) adopta una articulación estrecha entre fundamentos (y sus objetos curriculares generales) y conocimientos y habilidades. Sin embargo, el implementar el currículo no es posible usando solo los conocimientos y sus habilidades. Esto es radicalmente nuevo. La formulación de objetos y ejes curriculares transversales a todas las áreas matemáticas, de entrada, rompe cualquier intento por seguir una aproximación basada solo en contenidos.

Este currículo constituyó una "puntilla" final a las ideas de la New Math (por ejemplo, en virtud del uso de la teoría de conjuntos, o con la visión "formalista" sobre la naturaleza de las matemáticas que nutrió aquella reforma), pero además una ruptura con otras visiones (paradigmas) que estuvieron presentes durante muchas décadas en la educación nacional. Se trata de un currículo coherente con la investigación y buenas prácticas de la Educación Matemática en el mundo, aunque mediante construcciones teóricas originales. Es un "salto cuántico" (Ruiz, 2020, p. 11) o con Artigue (2018): una fuerte "perturbación ecológica" en el sistema educativo. La consecuencia ha sido y será, inevitablemente, la dificultad para su implementación.

El MEP-DM (2018) sintetiza bien cómo en la segunda década del siglo XXI se avanzó en la implantación de los nuevos programas. Se dieron muchas acciones, las cuales incluyeron: una instalación gradual (programas de transición), planes piloto, soporte documental, formación continua y asesoría (cursos presenciales y bimodales, asesoramientos regionales, MOOC's, Mini-MOOC's, recientemente Recursos Libres de Matemáticas), materiales de apoyo para estudiantes, y, a subrayar, múltiples recursos con un uso innovador y fuerte de tecnologías (Ruiz, 2020). Todo a la medida del currículo. Y mucho de ello nutrido por el Proyecto Reforma de la Educación Matemática en Costa Rica del MEP (www.reformamatematica.net).

También cabe señalar que avanzaron ajustes positivos en las pruebas nacionales. Las universidades públicas, en convergencia, fueron ajustando programas y acciones al nuevo currículo, con el fin de generar soportes mediante su acción social, investigación y docencia, para así contribuir a la Reforma Matemática. En fin, la penetración del currículo, aunque desigual y lentamente, progresaba en las diversas regiones y espacios del sistema educativo (MEP-DM, 2018).

Desde la segunda mitad de 2018, sin embargo, el escenario cambió. Una huelga extendida por tres meses y la situación provocada por la pandemia de la COVID-19 han generado una crisis educativa colosal. Entre las muchas dimensiones que se pueden invocar aquí, hay una que es vital: la pérdida de escolaridad que se ha ocasionado (y la que aún se dará). Esto expone al país a un periodo largo y muy complicado para avanzar con los objetivos educativos. Históricamente, hay un antes y un después de esta crisis.

En este contexto, la Reforma Matemática se vuelve más compleja. Lo que ya a priori era un plan de largo plazo, ahora exige mucho más tiempo, dedicación y, sobre todo, enfoques renovados para poder apenas encontrar estabilidad y progreso. ¿Qué significa esta pérdida de escolaridad en la asignatura que carga con el síndrome de la "matefobia"? Ausencia en la población estudiantil de muchos conocimientos 
matemáticos, habilidades y poco desarrollo de capacidades cognitivas superiores. Se acumularán varios años perdidos, aunque cuántos dependerá de la situación socioeconómica y familiar, de la región, y el contexto educativo. Es un retroceso diversificado a nivel social. Las diferencias y las brechas inevitablemente se potenciarán.

Para abordar esta problemática expuesta, el país requiere estrategias múltiples donde se invocan diversas instancias. También se apunta a las universidades formadoras de docentes. En primer lugar, la preparación docente deberá ajustarse para incorporar a un nuevo perfil de estudiantes con diversos niveles de escolaridad, algunos muy débiles (aunque hayan logrado completar formalmente la secundaria). En segundo término, esta formación inicial deberá desarrollar competencias para que sus egresados puedan participar con éxito en un contexto diferente afectado por condiciones distintas a las que se tenían hace poco tiempo, un escenario que durará muchos años. Y aquí hacemos una propuesta decisiva: no solo se plantean ajustes en el papel de las tecnologías virtuales que han transformado mucho los quehaceres educativos (educación híbrida, virtualización de procesos, "aulas invertidas" y fuerte uso de internet). Es claro que las competencias digitales docentes que han cultivado las instituciones formadoras no han sido suficientes. La pandemia lo ha evidenciado. Ajustes en este territorio son muy importantes. Sin embargo, se debe tener una visión aún más amplia: los contenidos, las competencias y los propósitos de estos programas deben concebirse de otra manera. El grave faltante de conocimientos y capacidades matemáticas de los nuevos estudiantes obliga a novedosas estrategias que además deben ser diferenciadas.

Por otra parte, los nuevos egresados deberán lidiar con contextos dispares y con fuertes rezagos en escolaridad. Esto ya es sumamente complejo, pero no debe olvidarse además que el escenario que vivimos obliga a convivir con la incertidumbre. ¿Cómo enseñar en un medio tan disperso, desigual e incierto? ¿Cómo interpretar el currículo nacional para avanzar en ese escenario? ¿Cuáles estrategias y metodologías de mediación tomando en cuenta por supuesto la participación radicalmente nueva de competencias tecnológicas?

No es un desafío sencillo para el ajuste curricular. Hay, sin embargo, características de nuestros programas de matemáticas que favorecen las soluciones; por ejemplo: una mediación centrada en capacidades y no solo en listados de conocimientos, y una integración vertical y horizontal de todos los objetos curriculares. La contextualización es otro soporte clave. El papel visionario de las tecnologías se añade. Los recursos ya construidos en la Reforma Matemática son otro soporte. Como en toda crisis, también surgen oportunidades. Una regla de oro: la flexibilidad, en el rediseño de estos programas resultará ahora más importante que nunca.

\section{SÍNTESIS Y REFLEXIÓN FINAL}

La perspectiva histórica que nos hacía prever tiempos "generacionales" en la Reforma Matemática, en el actual escenario nos apunta hacia la necesidad de ajustes significativos de los programas de formación docente para apoyar su derrotero positivo. Esto no es para un futuro abstracto y lejano, sino para esta tercera década del siglo XXI, en la que ya vivimos. Es decisivo que las universidades comprendan esta urgencia. 


\section{REFERENCIAS}

Artigue, M. (2018). Implementing curricular reforms: a systemic challenge. En Y. Shimizu y R. Vithal (Eds.), School Mathematics Curriculum Reforms: Challenges, Changes and Opportunities: Proceedings of ICMI Study 24 Conference. Tsukuba, Japan: ICMI.

Barrantes, H. y Ruiz, A. (1995). 1964. En A. Ruiz (Ed.), Historia de las Matemáticas en Costa Rica. Una introducción. Costa Rica: Editoriales de la Universidad de Costa Rica y Universidad Nacional.

Ministerio de Educación Pública de Costa Rica MEP (2012). Programas de estudio de Matemáticas I y II Ciclo de la Educación Primaria, III Ciclo de Educación General Básica y Educación Diversificada. San José, Costa Rica: autor.

Ministerio de Educación Pública, Despacho de la Ministra MEP-DM (2018). Programas oficiales de Matemáticas. Informe de implementación 2012-2017. Costa Rica: autor. https://www.reformamatematica.net/wp-content/uploads/2019/09/Informe-MEP_Reforma-Matematica-hasta-2017.pdf

Ruiz, A. (2018). Evaluación y pruebas nacionales para un currículo de matemáticas que enfatiza capacidades superiores. México: Comité Interamericano de Educación Matemática.

Ruiz, A. (2020). Reforma Matemática en tiempos de crisis nacional: fortalezas, debilidades, amenazas, oportunidades. Cuadernos de Investigación y Formación en Educación Matemática, 19:9-53. https://revistas.ucr.ac.cr/index.php/cifem/article/view/45218/45173 